\title{
Quercetin suppresses inflammatory cytokine production in rheumatoid arthritis fibroblast-like synoviocytes
}

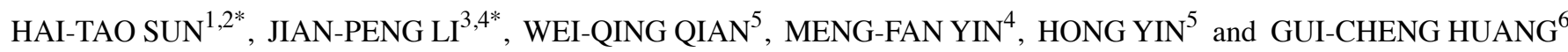 \\ ${ }^{1}$ Laboratory of New Techniques of Restoration and Reconstruction of Orthopedics and Traumatology, \\ Nanjing University of Chinese Medicine, Nanjing, Jiangsu 210023; ${ }^{2}$ Department of Emergency Trauma Center, \\ Wuxi Huishan District People's Hospital, Wuxi, Jiangsu 214187; ${ }^{3}$ Department of Orthopedics, Tianjin Medical University, \\ Tianjin 300070; ${ }^{4}$ Department of Orthopedics, Tianjin Fifth Central Hospital, Tianjin 300450; ${ }^{5}$ Department of Orthopedics, \\ The Third Affiliated Hospital of Nanjing University of Chinese Medicine, Nanjing, Jiangsu 210001; \\ ${ }^{6}$ Laboratory of New Techniques of Restoration and Reconstruction of Orthopedics and Traumatology, \\ The First Clinical Medical College, Nanjing University of Chinese Medicine, Nanjing, Jiangsu 210023, P.R. China
}

Received June 17, 2020; Accepted June 11, 2021

DOI: $10.3892 / \mathrm{etm} .2021 .10695$

\begin{abstract}
Rheumatoid arthritis (RA) is a chronic, progressive and systemic autoimmune disease mainly characterized by symmetric multijoint synovitis. Quercetin has anti-inflammatory, anti-oxidation and immune regulation activities, and therefore shows high medicinal value. The present study aimed to observe the effect of quercetin on fibroblast-like synoviocytes (FLSs) in RA. Rheumatoid arthritis fibroblast-like synoviocytes (RAFLSs) were pretreated with $50 \mathrm{nmol} / \mathrm{l}$ quercetin for $2 \mathrm{~h}$ and were then stimulated using TNF- $\alpha$ for $24 \mathrm{~h}$ for subsequent experiments. RAFLSs were transfected with short interfering (si)-X-inactive specific transcript (XIST), microRNA (miR)-485 mimic, miR-485 inhibitor or si-PSMB8 or combination. ELISA, PCR and western blotting was used to evaluate the effect of quercetin on RAFLSs treated with TNF- $\alpha$. It was revealed that quercetin inhibited the production of inflammatory cytokines and the expression of XIST in RAFLSs induced by TNF- $\alpha$. Bioinformatics analysis indicated that XIST acted as a sponge for miR-485 and that proteasome subunit $\beta$ type-8 (PSMB8) was a direct target of miR-485. Moreover, PSMB8 functioned as a suppressor in inflammatory cytokine production of RAFLSs induced by TNF- $\alpha$. Overall, quercetin was observed to inhibit the production
\end{abstract}

Correspondence to: Dr Gui-Cheng Huang, Laboratory of New Techniques of Restoration and Reconstruction of Orthopedics and Traumatology, The First Clinical Medical College, Nanjing University of Chinese Medicine, 155 Hanzhong Road, Nanjing, Jiangsu 210023, P.R. China

E-mail: hgc_njcmu@126.com

*Contributed equally

Key words: rheumatoid arthritis, quercetin, $\mathrm{X}$-inactive specific transcript, microRNA-485, proteasome subunit $\beta$ type- 8 of inflammatory cytokines and the expression of XIST in RAFLSs induced by TNF- $\alpha$. Moreover, XIST-silencing could suppress the inflammatory reaction by sponging miR-485 in cells treated with TNF- $\alpha$. Altogether, quercetin could suppress the development of RA in vitro.

\section{Introduction}

Rheumatoid arthritis (RA) is a common inflammatory disease characterized by inflammatory cell infiltration that leads to degeneration of the synovium, articular cartilage and bone (1). The occurrence and development of RA involves the release of a large number of inflammatory cytokines and mediators, which causes irreversible destruction of cartilage and bone in the joint (2). With the increase of an ageing population in human society, the incidence of RA has shown an increased trend in developed countries from 1995 to $2001(3,4)$. Currently, RA treatment relies on non-steroidal anti-inflammatory drugs, glucocorticoids, methotrexate and other symptomatic methods of controlling the disease. These drugs can improve the symptoms of RA, but long-term use has strong toxic side effects, such as inhibiting the immune function of the body (5). Therefore, finding safe and effective drugs for the treatment of $\mathrm{RA}$ is of great importance.

Quercetin is the most important bioflavonoid in the human diet, and is widely found in the flowers, leaves and fruits of plants $(6,7)$. Quercetin has anti-inflammatory, anti-oxidation and immune regulation activities $(8,9)$, and therefore shows high medicinal value. Clinical studies have shown that quercetin has a good effect on relieving knee osteoarthritis, as well as rheumatoid joint and body pain after exercise (10-12). Fibroblast-like synoviocytes (FLSs) are the main effector cells and can release a variety of inflammatory cytokines, aggravating the progression of RA $(13,14)$. In addition, TNF- $\alpha$ can promote the activation and bone resorption of osteoclasts, and can induce the expression of other inflammatory factors, adhesion molecules and proteases associated with bone and cartilage; thus, causing the thickening of the synovial lining 
layer and further exacerbating the inflammatory response of the joints (15). The present study therefore aimed to observe the effect of quercetin on FLSs in RA and explore the possible mechanism of quercetin in the treatment of RA.

\section{Materials and methods}

Cell culture. The primary cells RAFLSs were attained from The Cell Bank of the Type Culture Collection of The Chinese Academy of Sciences (cat. no. 408-05a). All cells were maintained in DMEM (Invitrogen; Thermo Fisher Scientific, Inc.) containing 10\% FBS (HyClone; Cytiva) $100 \mathrm{U} / \mathrm{ml}$ penicillin and $100 \mu \mathrm{g} / \mathrm{ml}$ streptomycin in an incubator at $37^{\circ} \mathrm{C}$. After culturing for $24 \mathrm{~h}$, RAFLSs were pretreated with $50 \mathrm{nmol} / 1$ quercetin (MilliporeSigma) for $2 \mathrm{~h}$ at $37^{\circ} \mathrm{C}$ and then were stimulated using $50 \mathrm{ng} / \mathrm{ml} \mathrm{TNF}-\alpha$ for $24 \mathrm{~h}$ at $37^{\circ} \mathrm{C}$ for the following experiments.

Cell transfection. Small interfering (si)-RNAs targeting XIST (si-XIST; final concentration, $20 \mathrm{nM}$ ) and its scrambled negative control (NC) siRNA (final concentration, $20 \mathrm{nM}$ ), miR-485 inhibitor (final concentration, $30 \mathrm{nM}$ ) and scrambled inhibitor control (miR-NC; final concentration, $30 \mathrm{nM}$ ) and miR-485 mimics (final concentration, $15 \mathrm{nM}$ ) and scrambled mimic control (miR-NC mimics; final concentration, $15 \mathrm{nM}$ ) were obtained from Shanghai GenePharma Co., Ltd. A total of $1 \times 10^{6}$ RAFLSs were plated into six-well plates for $12 \mathrm{~h}$, then the constructs were transfected into RAFLSs by mixing with Lipofectamine ${ }^{\circledR} 2000$ reagent (Invitrogen; Thermo Fisher Scientific, Inc.) at $37^{\circ} \mathrm{C}$ for $15 \mathrm{~min}$ following the manufacturer's instructions. A total of 24-72 $\mathrm{h}$ after transfection, the transfected cells were collected for subsequent experiments. The sequences of the transfected molecules were as follows: si-XIST, 5'-GCAAAUGAA AGCUACCAAU-3'; si-NC, 5'-AAUUCUCCGAACGUGUCACGU-3'; miR-485 inhibitor, 5'-UCACGCGAGCCGAACGAACAAA-3'; anti-miR-NC, 5'-CAGUACUUUUGUGUAGUACAAA-3'; miR-485 mimic, 5'-GUCAUACACGGCUCUCCUCUCU-3'; miR-NC, 5'-UUC UCCGAACGUGUCACGUTT-3'.

Enzyme-linked immunosorbent assay (ELISA). The concentration of inflammatory cytokines in transfected and non-transfected RAFLSs (IL-1 $\beta$, IL-6 and IL-8) was determined using ELISA kits (cat. nos. KE00021, KE00139 and KE00006; Proteintech Group, Inc.) following the manufacturer's protocol. A microplate reader was used to read the absorbance at $450 \mathrm{~nm}$.

Reverse transcription-quantitative $(R T-q) P C R$ analysis. Total cellular RNAs were extracted from transfected and non-transfected RAFLSs using the TRIzol ${ }^{\circledR}$ reagent (Invitrogen; Thermo Fisher Scientific, Inc.). cDNA synthesis was performed using Maxima Probe qPCR Master mix (Fermentas; Thermo Fisher Scientific, Inc.) according to the manufacturer's instructions. Quantitative analysis of XIST and PSMB8 expression was performed using SYBR ${ }^{\circledR}$ Premix Ex $\mathrm{Taq}^{\mathrm{TM}}$ reagent (Takara Bio, Inc.) and GAPDH was used as the endogenous control. Thermocycling conditions were as follows: $94^{\circ} \mathrm{C}$ for $3 \mathrm{~min}$; 40 cycles of $94^{\circ} \mathrm{C}$ for $30 \mathrm{sec}, 55^{\circ} \mathrm{C}$ for $30 \mathrm{sec}$ and $72^{\circ} \mathrm{C}$ for $1 \mathrm{~min}$; and $72^{\circ} \mathrm{C}$ for $10 \mathrm{~min}$. The expression level of miR-485 was analyzed using SYBR ${ }^{\circledR}$ PrimeScript $^{\mathrm{TM}}$ miRNA RT-PCR kit (Takara Biotechnology Co., Ltd.) and U6 small nuclear RNA was used as the internal reference. Thermocycling conditions were as follows: Denaturation at $95^{\circ} \mathrm{C}$ for $10 \mathrm{sec} ; 40$ cycles of $95^{\circ} \mathrm{C}$ for $5 \mathrm{sec}$ and $60^{\circ} \mathrm{C}$ for $20 \mathrm{sec}$; followed by dissociation curve analysis at $95^{\circ} \mathrm{C}$ for $60 \mathrm{sec}$, $55^{\circ} \mathrm{C}$ for $30 \mathrm{sec}$ and $95^{\circ} \mathrm{C}$ for $30 \mathrm{sec}$. Primers were purchased from Sangon Biotech Co., Ltd., and primer sequences were as follows: GAPDH Forward, 5'-CTTTGGTATCGTGGA AGGACTC-3' and reverse, 5'-GTAGAGGCAGGGATGATG TTCT-3'; U6 forward, 5'-GCTTCGGCAGCACATATACTA AAAT-3' and reverse, 5'-CGCTTCACGAATTTGCGTGTC AT-3'; lncRNA XIST forward, 5'-AATGGAACGGGCTGA GTTTTAC-3' and reverse, 5'-TCATCCGCTTGCGTTCAT AG-3'; miR-485 forward, 5'-CAGAGGCTGGCCGTGAT-3' and reverse, 5'-GTCGTATCCAGTGCAGGGTCCGAGGTA TTCGCACTGGATACGACGAATTC-3'; PSMB8 forward, 5'-GCTGCCTTCAACATAACATCA-3' and reverse, 5'-CTG CCACCACCACCATTA-3'. The relative expression was analysis using $2^{-\Delta \Delta \mathrm{Cq}}(16)$.

Western blotting. Total protein was isolated from RAFLSs using pre-cold RIPA lysis buffer (Beyotime Institute of Biotechnology) including protease inhibitor. Total protein was quantified using a BCA protein assay kit (Pierce; Thermo Fisher Scientific, Inc.). A total of $30 \mu \mathrm{g}$ protein sample per lane were separated by $10 \%$ SDS-PAGE, and subsequently transferred onto a nitrocellulose membrane (MilliporeSigma). The membranes were blocked with $5 \%$ non-fat milk at room temperature for $1 \mathrm{~h}$. Subsequently, the membranes were incubated overnight at $4^{\circ} \mathrm{C}$ with primary antibodies targeted against: PSMB8 (1:1,000; cat. no. ab180606) and GAPDH (1:1,000; cat. no. ab181602) (all from Abcam). The membranes were incubated with a horseradish peroxidase-labeled anti-rabbit IgG secondary antibody (1:1,000; sc-2027; Santa Cruz Biotechnology, Inc.) at room temperature for $1 \mathrm{~h}$. Protein bands were visualized using an ECL detection kit (Pierce; Thermo Fisher Scientific, Inc.) and analyzed with Quantity One v4.6.2 software (Bio-Rad Laboratories, Inc.). GAPDH was used as the loading control.

Luciferase reporter assay. The XIST fragments and PSMB8 3'-untranslated regions containing wild-type (WT) or mutant (MUT) miR-485 binding sites were attained from Shanghai GenePharma Co., Ltd. They were cloned into the firefly luciferase gene reporter vector pmiRGLO (Promega Corporation). The plasmid was synthesized by Invitrogen; Thermo Fisher Scientific, Inc. Then $48 \mathrm{~h}$ after transfection using Lipofectamine ${ }^{\circledR} 2000$ reagent (Invitrogen; Thermo Fisher Scientific, Inc.) at $37^{\circ} \mathrm{C}$ with $5 \% \mathrm{CO}_{2}$, the luciferase assay was performed using the dual-luciferase reporter assay system kit (Promega Corporation) according to the manufacturer's instructions. The luciferase activity was analyzed using a Modulus single-tube multimode reader (Promega Corporation) in comparison with Renilla luciferase activity. The following sequences were used: XIST-WT Forward, 5'-CGCCAGCTTCGACAATTCGAC-3', and reverse, 5'-ACT TGAAGCGGCCATACGTTGACAA-3'; XIST-MUT forward, 5'-TCAAAACCCAACTAGAGGACGATTTGTGA-3', and reverse, 5'-AACCCAGAAAACCGTACGGCAATCGT-3'; 

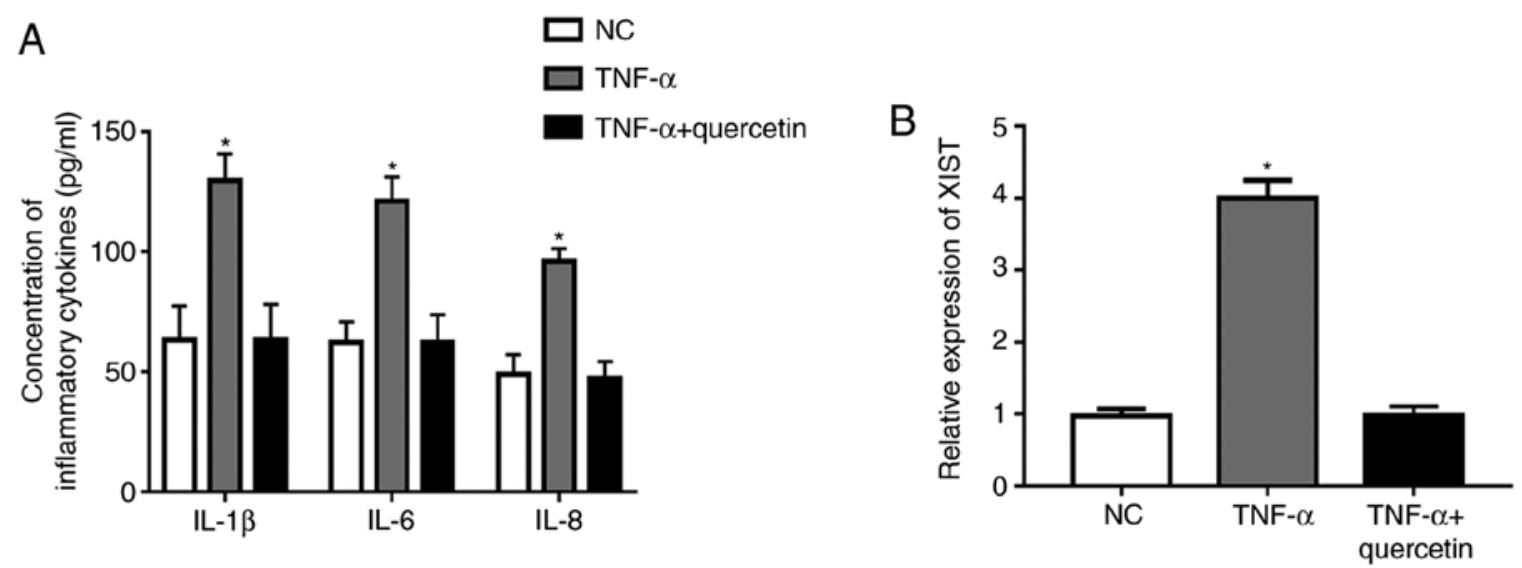

Figure 1. Quercetin inhibits the expression level of XIST and the production of inflammatory cytokines in FLSs induced by TNF- $\alpha$. (A) Under TNF- $\alpha$ stimulation, the concentrations of inflammatory cytokines (IL-1 $\beta$, IL-6 and IL-8) were increased in RAFLSs, which was suppressed by quercetin. (B) Under TNF- $\alpha$ stimulation, the expression level of XIST was significantly upregulated in RAFLSs, which was suppressed by quercetin. ${ }^{*} \mathrm{P}<0.05$ vs. NC group. XIST, X-inactive specific transcript; RAFLSs, rheumatoid arthritis fibroblast-like synoviocytes; NC, negative control.

PSMB8-WT forward, 5'-CCGCTAAGTCCGAGTAGTCA-3', and reverse, 5'-ATAAGAATGCGGCCGAATTCATGG GAA-3'; and PSMB8-MUT forward, 5'-TTAGGACACGCA TCGGATTACGCTATTAT-3', and reverse, 5'-GACACCTGT TTACGGCTACCCGCAAATATGCCAA-3'.

Bioinformatics analysis. The binding sites between miR-485 and XIST, as well as miR-485 and PSMB8 were predicted by StarBase v2.0 (http://www.microrna.gr/lncBase) or TargetScan software v7.1 (http://www.targetscan.org/vert_71/), respectively.

Statistical analysis. All measured data are expressed as the mean \pm standard deviation. Differences were calculated with paired Student's t-test for comparisons between two groups or one-way ANOVA followed by Dunnett's or Tukey's post hoc test for multiple comparisons. All statistical analyses were performed using GraphPad Prism 5.0 (GraphPad Software, Inc.) and SPSS 14.0 (SPSS, Inc.). $\mathrm{P}<0.05$ was considered to indicate a statistically significant difference. Experiments were repeated three times.

\section{Results}

Quercetin inhibits the production of inflammatory cytokines and the expression of XIST in RAFLSs induced by TNF- $\alpha$. Under TNF- $\alpha$ stimulation, the concentration of inflammatory cytokines (IL-1 $\beta$, IL-6 and IL-8) and the expression level of XIST were significantly upregulated in RAFLSs compared with those in the NC (Fig. 1A and B). However, treatment with quercetin could markedly suppress the promotion of TNF- $\alpha$ in the production of inflammatory cytokines, and the expression level of XIST was markedly decreased in the FLSs (Fig. 1A and B). These results indicated that quercetin could suppress the inflammation reaction in RAFLSs induced by TNF- $\alpha$.

$X I S T$ acts as a sponge for $m i R-485$. Subsequently, the potential molecular mechanisms were explored and the possible targets of XIST were predicted using the StarBase database v2.0.
The bioinformatics analysis indicated that miR-485 had a potential binding site with the XIST sequence (Fig. 2A). Transfection with the miR-485 mimic was demonstrated to upregulate the expression of miR-485 in RAFLSs (Fig. 2B). Meanwhile, the miR-485 mimic markedly repressed luciferase activity in the XIST wild-type compared with that in the NC (Fig. 2C). TNF- $\alpha$ could significantly downregulate the expression level of miR-485 in RAFLSs, which was rescued by quercetin (Fig. 2D). XIST was significantly underexpressed in RAFLSs after transfection with si-XIST (Fig. 2E). In addition, XIST-silencing significantly promoted the expression of miR-485, which was reversed by the miR-485 inhibitor in RAFLSs treated with TNF- $\alpha$ (Fig. 2F). These results indicated that XIST interacted with miR-485.

PSMB8 is a direct target of miR-485. According to TargetScan analysis, a highly conserved putative binding site with miR-485 was identified at the PSMB8 3'-untranslated region (Fig. 3A). The miR-485 mimic significantly repressed the fluorescence in the PSMB8 wild-type compared with that in the NC (Fig. 3B). TNF- $\alpha$ could significantly upregulate the expression level of PSMB8 in RAFLSs, which was reversed by quercetin (Fig. 3C). The miR-485 inhibitor could significantly downregulate the expression level of miR-485 in RAFLSs (Fig. 3D). In addition, the expression of PSMB8 was significantly decreased in RAFLSs after transfection with si-PSMB8 (Fig. 3E). miR-485 inhibitor could significantly enhance the expression of PSMB8 at the mRNA and protein levels, which was abolished by si-PSMB8 in RAFLSs treated with TNF- $\alpha$ (Fig. 3F). These results indicated that miR-485 directly targeted PSMB8.

PSMB8 functions as a suppressor in the production of inflammatory cytokines in RAFLSs induced by TNF- $\alpha$. After transfection with si-PSMB8, the concentrations of inflammatory cytokines (IL-1 $\beta$, IL-6 and IL-8) were significantly decreased in transfected RAFLSs treated with TNF- $\alpha$ compared with the untransfected cells treated with TNF- $\alpha$ (Fig. 4A). Furthermore, quercetin suppressed the inflammatory reaction in RAFLSs under TNF- $\alpha$ stimulation, which 
A

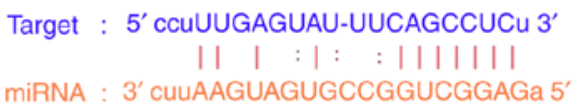

C
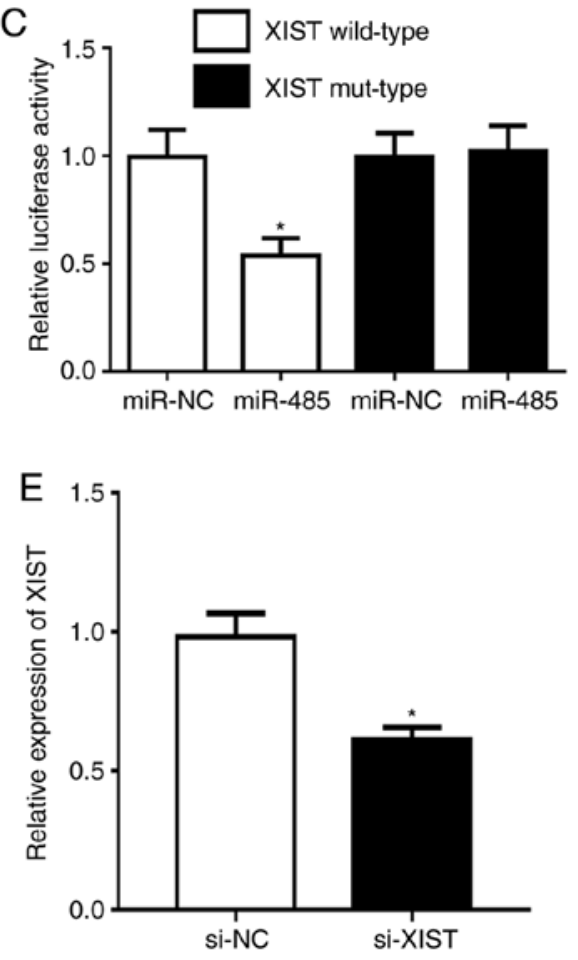

B

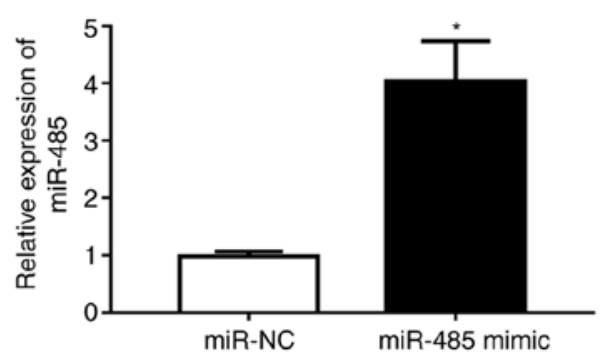

$\mathrm{D}$

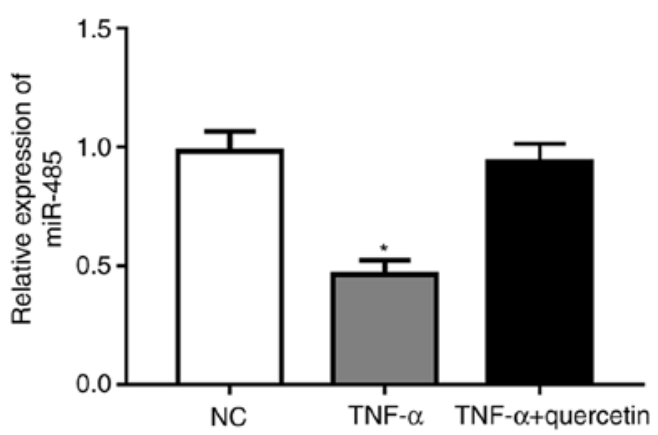

$\mathrm{F}$

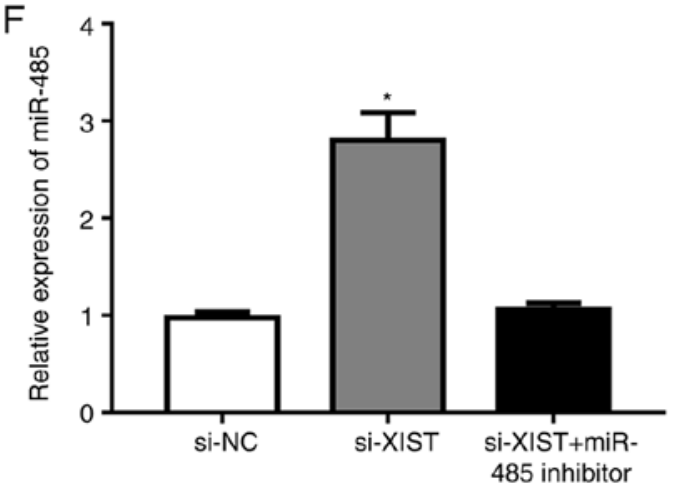

Figure 2. XIST acts as a sponge for miR-485. (A) Potential binding site of miR-485 with the XIST sequence. (B) miR-485 mimic upregulated the expression of miR-485 in RAFLSs. (C) Luciferase activity reporter assays demonstrated that the miR-485 mimic inhibited luciferase activity in the XIST wild-type. (D) TNF- $\alpha$ could downregulate the level of miR-485 expression in RAFLSs, which was rescued by quercetin. (E) The expression of XIST was significantly downregulated in RAFLSs transfected with si-XIST. (F) XIST-silencing effectively promoted the expression of miR-485, which was abolished by miR-485 inhibitor in RAFLSs treated with TNF- $\alpha$. ${ }^{*} \mathrm{P}<0.05$ vs. NC group. XIST, X-inactive specific transcript; miR/miRNA, microRNA; NC, negative control; si-, short interfering; mut; mutant; RAFLSs, RA fibroblast-like synoviocytes.

was rescued by transfection with si-PSMB8 (Fig. 4B). These results indicated that PSMB8 could suppress the inflammation reaction in RAFLSs induced by TNF- $\alpha$.

\section{Discussion}

As a natural active product from plants, quercetin is a secondary metabolite isolated and extracted from fagaceae plants by natural medicinal chemistry (17). In previous years, quercetin has been verified to inhibit the inflammatory response in a number of diseases, including malignant tumors (18), diabetic mellitus (19) and atherosclerosis (20). The present study assessed the role of quercetin on the development of RA. It is hypothesized that once fibroblasts are activated in the pathogenesis of RA, a series of inflammatory factors and extracellular matrix degrading enzymes will be produced to mediate the inflammatory response, causing the progressive destruction of bone and cartilage, and influencing the quality of life severely $(21,22)$. The present study observed that quercetin suppressed the inflammatory cytokine production of FLSs induced by TNF- $\alpha$.

As a class of non-coding transcripts, lncRNAs are $>200$ nucleotides in length and regulate gene expression at the epigenetic, transcriptional and post-transcriptional levels $(23,24)$. IncRNA XIST was originally identified in the regulation of $\mathrm{X}$ inactivation in the female mammal somatic cell (25). Previous research suggests that deregulated expression of XIST is involved in the inflammatory reaction of some diseases including inflammatory neuralgia and acute pneumonia $(26,27)$. Consistent with previous research, the present study found that the expression level of XIST was upregulated in FLSs induced by TNF- $\alpha$, which was abolished by quercetin. IncRNAs can regulate gene expression by sponging miRNAs (28). Bioinformatics analysis indicated that miR-485 had a potential binding site with the XIST sequence. In addition, the level of miR-485 expression was 


\section{A Target: 5' uauUUCAGUGUCGGCAGCCUCc 3' ||$|:::|||||||$ miRNA : 3' cuUAAGUAGUGCCGGUCGGAGa 5'}
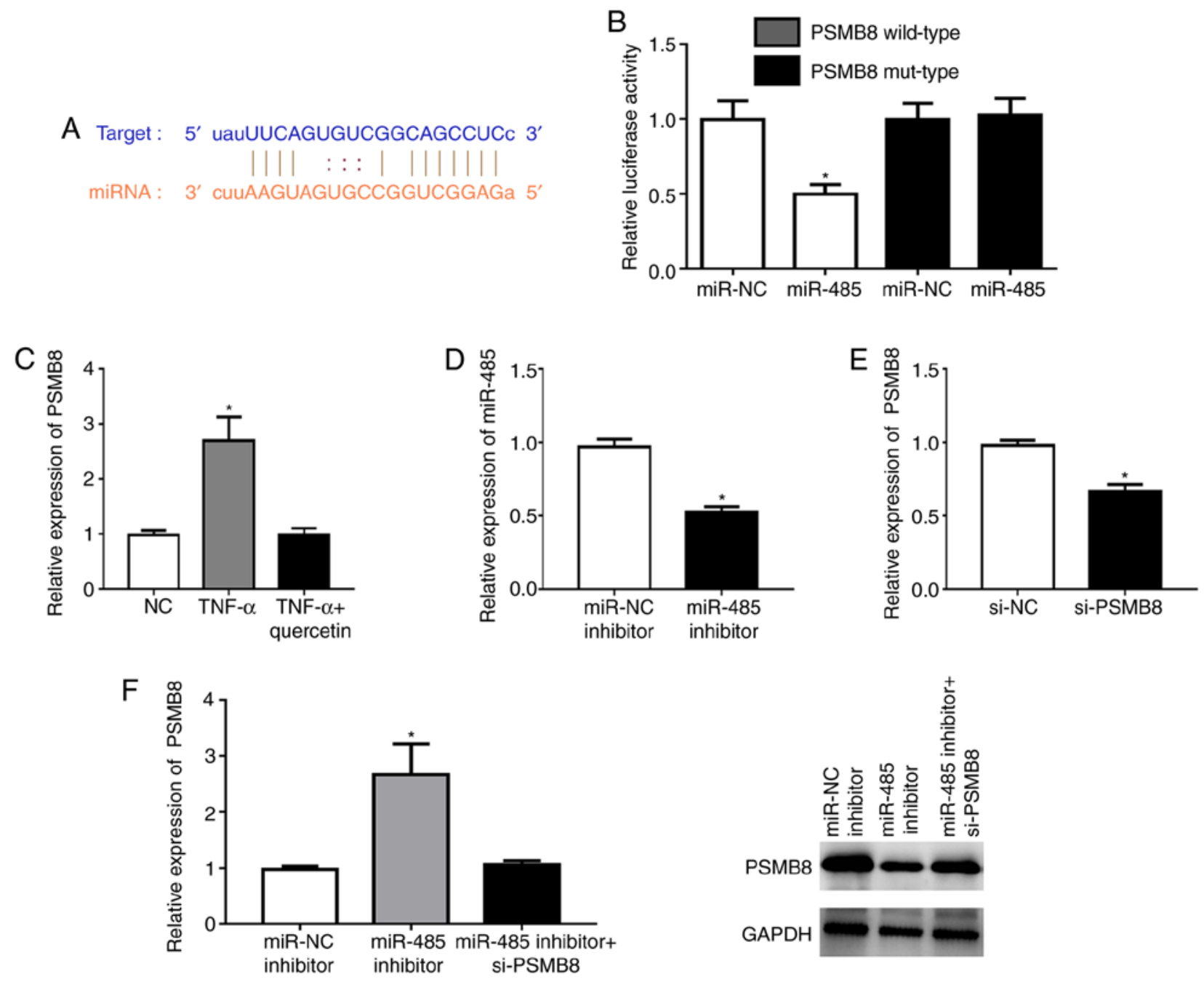

Figure 3. PSMB8 is a direct target of miR-485. (A) A highly conserved putative binding site with miR-485 was identified at the PSMB8 3'-untranslated region. (B) miR-485 mimic could repress the fluorescence in the PSMB8 wild-type. (C) TNF- $\alpha$ could significantly upregulate the level of PSMB8 expression in RAFLSs, which was reversed by quercetin. (D) miR-485 inhibitor could downregulate the expression of miR-485 in RAFLSs. (E) Expression of PSMB8 was significantly decreased in RAFLSs after transfection with si-PSMB8. (F) miR-485 inhibitor could significantly increase PSMB8 expression at the mRNA and protein levels, which was abolished by si-PSMB8 in RAFLSs treated with TNF- $\alpha$. ${ }^{\mathrm{P}}<0.05$ vs. NC group. PSMB8, proteasome subunit $\beta$ type- 8 ; miR/miRNA, microRNA; si-, short interfering; NC, negative control; RAFLSs, rheumatoid arthritis fibroblast-like synoviocytes.
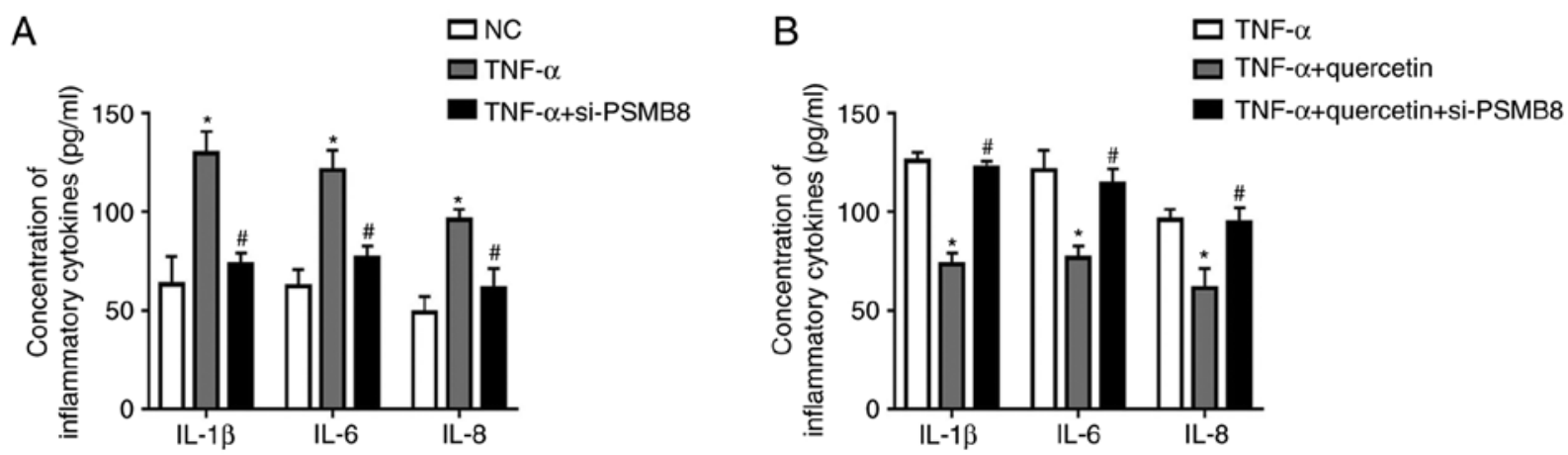

Figure 4. PSMB8 functions as a suppressor in inflammatory cytokine production of RAFLSs induced by TNF- $\alpha$. (A) After transfection with si-PSMB8, the concentration of inflammatory cytokines (IL-1 $\beta$, IL-6 and IL-8) was decreased in RAFLSs treated with TNF- $\alpha$. (B) Quercetin did not suppress the inflammation reaction in RAFLSs treated with si-PSMB8 under TNF- $\alpha$ stimulation. ${ }^{*} \mathrm{P}<0.05$ vs. NC group; ${ }^{*} \mathrm{P}<0.05$ vs. TNF- $\alpha$ group. PSMB8, proteasome subunit $\beta$ type-8; RAFLSs, RA fibroblast-like synoviocytes; si-, short interfering; NC, negative control

downregulated in FLSs treated with TNF- $\alpha$. Additionally, XIST-silencing effectively promoted miR-485 expression in cells after transfection.
miRNAs can target the 3'-untranslated region of mRNAs to control gene expression (29). The present study revealed that PSMB8 is a direct target of miR-485. PSMB8 can function as a 
subunit of the immunoproteasome to induce an inflammatory response as follows. Yamagishi (30) reported that specific inhibition of PSMB8 can decrease the secretion of inflammatory factors, thus delaying the inflammation response of diabetes mellitus. Koerner et al (31) revealed that the involvement of PSMB8 in the inflammatory response is closely associated with the development of colorectal cancer. The present study revealed that TNF- $\alpha$ could significantly upregulate the expression level of PSMB8 in RAFLSs. Furthermore, si-PSMB8 could inhibit the concentration of inflammatory cytokines in RAFLSs treated with TNF- $\alpha$. In addition, quercetin could not suppress the inflammatory reaction in RAFLSs treated with si-PSMB8 under TNF- $\alpha$ stimulation. These data suggested that PSMB8 functions as a suppressor in the production of inflammatory cytokines in RAFLSs induced by TNF- $\alpha$.

The present study observed that the suppression of inflammatory cytokine production by quercetin in FLSs induced by TNF- $\alpha$ is promising in vitro. However, the effect in vivo and the effect of pharmacokinetics and penetration of the synovial membrane on quercetin have yet to be investigated. Thus, further studies are planned to attempt to study the effect of quercetin on RA in vivo.

In conclusion, the present study observed quercetin-inhibited production of inflammatory cytokines and XIST expression in RAFLSs induced by TNF- $\alpha$. Moreover, XIST-silencing could suppress the inflammatory reaction by sponging miR-485 in cells treated with TNF- $\alpha$. Altogether, quercetin could suppress the development of RA in vitro.

\section{Acknowledgements}

Not applicable.

\section{Funding}

No funding was received.

\section{Availability of data and materials}

The datasets used and/or analyzed during the present study are available from the corresponding author on reasonable request.

\section{Authors' contributions}

HTS and JPL designed experiments. WQQ and MFY performed experiments. HY analyzed the data. $\mathrm{GCH}$ performed the preliminary experiments and wrote the manuscript, JPL and GCH revised the manuscript. HS and $\mathrm{GCH}$ confirm the authenticity of all the raw data. All authors have read and approved the final manuscript.

\section{Ethics approval and consent to participate}

All patients gave informed consent and this study was approved by The Ethics Committee of Nanjing University of Chinese Medicine.

\section{Patient consent for publication}

Not applicable.

\section{Competing interests}

The authors declare that they have no competing interests.

\section{References}

1. Smolen JS, Aletaha D and McInnes IB: Rheumatoid arthritis. Lancet 388: 2023-2038, 2016.

2. Firestein GS: Evolving concepts of rheumatoid arthritis. Nature 423: 356-361, 2003.

3. Gabriel SE and Michaud K: Epidemiological studies in incidence, prevalence, mortality, and comorbidity of the rheumatic diseases. Arthritis Res Ther 11: 229, 2009.

4. Klareskog L, Catrina AI and Paget S: Rheumatoid arthritis. Lancet 373: 659-672, 2009.

5. Tłustochowicz M, Śliwczyński AM, Brzozowska M, Teter Z and Marczak M: Sequentiality of treatment in the rheumatoid arthritis drug programme in the years 2009-2014. Arch Med Sci 14: 569-571, 2018.

6. Garcia-Mateos R, Aguilar-Santelises L, Soto-Hernández M and Nieto-Angel R: Flavonoids and antioxidant activity of flowers of Mexican Crataegus spp. Nat Prod Res 27: 834-836, 2013.

7. Harnly JM, Doherty RF, Beecher GR, Holden JM, Hay towitz DB, Bhagwat S and Gebhardt S: Flavonoid content of U.S. fruits, vegetables, and nuts. J Agric Food Chem 54: 9966-9977, 2006.

8. Boots AW, Wilms LC, Swennen EL, Kleinjans JC, Bast A and Haenen GR: In vitro and ex vivo anti-inflammatory activity of quercetin in healthy volunteers. Nutrition 24: 703-710, 2008.

9. McAnulty LS, Miller LE, Hosick PA, Utter AC, Quindry JC and McAnulty SR: Effect of resveratrol and quercetin supplementation on redox status and inflammation after exercise. Appl Physiol Nutr Metab 38: 760-765, 2013.

10. Mamani-Matsuda M, Kauss T, Al-Kharrat A, Rambert J, Fawaz F, Thiolat D, Moynet D, Coves S, Malvy D and Mossalayi MD: Therapeutic and preventive properties of quercetin in experimental arthritis correlate with decreased macrophage inflammatory mediators. Biochem Pharmacol 72: 1304-1310, 2006.

11. Natarajan V, Krithica N, Madhan B and Sehgal PK: Formulation and evaluation of quercetin polycaprolactone microspheres for the treatment of rheumatoid arthritis. J Pharm Sci 100: 195-205, 2011.

12. O'Fallon KS, Kaushik D, Michniak-Kohn B, Dunne CP, Zambraski EJ and Clarkson PM: Effects of quercetin supplementation on markers of muscle damage and inflammation after eccentric exercise. Int J Sport Nutr Exerc Metab 22: 430-437, 2012.

13. Bustamante MF, Garcia-Carbonell R, Whisenant KD and Guma M: Fibroblast-like synoviocyte metabolism in the pathogenesis of rheumatoid arthritis. Arthritis Res Ther 19: 110, 2017.

14. Mor A, Abramson SB and Pillinger MH: The fibroblast-like synovial cell in rheumatoid arthritis: A key player in inflammation and joint destruction. Clin Immunol 115: 118-128, 2005.

15. Davis JM III and Matteson EL; American College of Rheumatology; European League Against Rheumatism: My treatment approach to rheumatoid arthritis. Mayo Clin Proc 87: 659-673, 2012.

16. Livak KJ and Schmittgen TD: Analysis of relative gene expression data using real-time quantitative PCR and the 2(-Delta Delta C(T)) method. Methods 25: 402-408, 2001.

17. Wang LL, Jiang MX, Xu SX, Sun QS, Zeng GY and Zhou YJ: Two acylated flavonoid glycosides from the leaves of Quercus dentata. Nat Prod Commun 5: 1597-1599, 2010.

18. Lamson DW and Brignall MS: Antioxidants and cancer, part 3: Quercetin. Altern Med Rev 5: 196-208, 2000.

19. Fu J, Huang J, Lin M, Xie T and You T: Quercetin promotes diabetic wound healing via switching macrophages from M1 to M2 polarization. J Surg Res 246: 213-223, 2020.

20. Tribolo S, Lodi F, Connor C, Suri S, Wilson VG, Taylor MA, Needs PW, Kroon PA and Hughes DA: Comparative effects of quercetin and its predominant human metabolites on adhesion molecule expression in activated human vascular endothelial cells. Atherosclerosis 197: 50-56, 2008.

21. Firestein GS: Invasive fibroblast-like synoviocytes in rheumatoid arthritis. Passive responders or transformed aggressors? Arthritis Rheum 39: 1781-1790, 1996.

22. Zhang F, Zhang L and Zhang C: Long noncoding RNAs and tumorigenesis: Genetic associations, molecular mechanisms, and therapeutic strategies. Tumour Biol 37: 163-175, 2016. 
23. Geisler S and Coller J: RNA in unexpected places: Long non-coding RNA functions in diverse cellular contexts. Nat Rev Mol Cell Biol 14: 699-712, 2013.

24. Mattick JS and Rinn JL: Discovery and annotation of long noncoding RNAs. Nat Struct Mol Biol 22: 5-7, 2015.

25. Brown CJ, Ballabio A, Rupert JL, Lafreniere RG, Grompe M, Tonlorenzi R and Willard HF: A gene from the region of the human $\mathrm{X}$ inactivation centre is expressed exclusively from the inactive X chromosome. Nature 349: 38-44, 1991.

26. Sun W, Ma M, Yu H and Yu H: Inhibition of lncRNA X inactivate-specific transcript ameliorates inflammatory pain by suppressing satellite glial cell activation and inflammation by acting as a sponge of miR-146a to inhibit $\mathrm{Na}_{\mathrm{v}} 1.7$. J Cell Biochem 119: 9888-9898, 2018.

27. Zhang Y, Zhu Y, Gao G and Zhou Z: Knockdown XIST alleviates LPS-induced WI-38 cell apoptosis and inflammation injury via targeting miR-370-3p/TLR4 in acute pneumonia. Cell Biochem Funct 37: 348-358, 2019.

28. Zeisel MB, Druet VA, Wachsmann D and Sibilia J: MMP-3 expression and release by rheumatoid arthritis fibroblast-like synoviocytes induced with a bacterial ligand of integrin alpha5beta1. Arthritis Res Ther 7: R118-R126, 2005.
29. He L and Hannon GJ: MicroRNAs: Small RNAs with a big role in gene regulation. Nat Rev Genet 5: 522-531, 2004.

30. Yamagishi S: Role of advanced glycation end products (AGEs) and receptor for AGEs (RAGE) in vascular damage in diabetes. Exp Gerontol 46: 217-224, 2011.

31. Koerner J, Brunner T and Groettrup M: Inhibition and deficiency of the immunoproteasome subunit LMP7 suppress the development and progression of colorectal carcinoma in mice. Oncotarget 8: 50873-50888, 2017.

This work is licensed under a Creative Commons Attribution-NonCommercial-NoDerivatives 4.0 International (CC BY-NC-ND 4.0) License. 